\title{
On the Energy of Unitary Cayley Graphs
}

\author{
H.N. Ramaswamy and C.R. Veena \\ Department of Studies in Mathematics, University of Mysore \\ Manasagangothri, Mysore 570 006, INDIA \\ hnrama@gmail.com, veena_maths@rediffmail.com \\ Submitted: Mar 2, 2009; Accepted: Jul 17, 2009; Published: Jul 24, 2009 \\ Mathematics Subject Classification: 05C50
}

\begin{abstract}
In this note we obtain the energy of unitary Cayley graph $X_{n}$ which extends a result of $\mathrm{R}$. Balakrishnan for power of a prime and also determine when they are hyperenergetic. We also prove that $\frac{E\left(X_{n}\right)}{2(n-1)} \geq \frac{2^{k}}{4 k}$, where $k$ is the number of distinct prime divisors of $n$. Thus the ratio $\frac{E\left(X_{n}\right)}{2(n-1)}$, measuring the degree of hyperenergeticity of $X_{n}$, grows exponentially with $k$.
\end{abstract}

Keywords: Spectrum of a graph; Energy of a graph; Unitary Cayley graphs; Hyperenergetic graphs.

\section{Introduction}

Let $G$ be a simple finite undirected graph with $n$ vertices and $m$ edges and let $A=\left(a_{i j}\right)$ be the adjacency matrix of graph $G$. The eigenvalues $\lambda_{1}, \lambda_{2}, \ldots, \lambda_{n}$ of $A$, assumed in nonincreasing order, are the eigenvalues of the graph $G$ called the Spectrum of $G$ denoted by Spec $G$. If the distinct eigenvalues of $G$ are $\mu_{1}>\mu_{2}>\cdots>\mu_{s}$, and their multiplicities are $m\left(\mu_{1}\right), m\left(\mu_{2}\right), \ldots, m\left(\mu_{s}\right)$, then we write

$$
\operatorname{Spec} G=\left(\begin{array}{cccc}
\mu_{1} & \mu_{2} & \ldots & \mu_{s} \\
m\left(\mu_{1}\right) & m\left(\mu_{2}\right) & \ldots & m\left(\mu_{s}\right)
\end{array}\right) .
$$

Spec $G$ is independent of labelling of the vertices of $G$. As $A$ is a real symmetric matrix with zero trace, these eigenvalues are real with sum equal to zero.

The energy $\mathbf{E}(\mathbf{G})$ of $G$ was defined by I. Gutman [6] in 1978 as the sum of the absolute values of its eigenvalues. 
Since the energy of a graph is not affected by isolated vertices, we assume throughout that graphs have no isolated vertices implying, in particular, that $m \geq \frac{n}{2}$. If a graph is not connected, its energy is the sum of the energies of its connected components. Thus there is no loss in generality in assuming that graphs are connected.

The complete graph $K_{n}$ has simple eigenvalue $n-1$ and eigenvalue -1 of multiplicity $n-1$. Thus its energy is given by $E\left(K_{n}\right)=2(n-1)$. The graph $G$ of order $n$ whose energy satisfies $E(G)>2(n-1)$ is called hyperenergetic and graph with energy $E(G) \leq 2(n-1)$ is called non-hyperenergetic.

The Line graph $L(G)$ of a graph $G$ is constructed by taking the edges of $G$ as vertices of $L(G)$, and joining two vertices in $L(G)$ whenever the corresponding edges in $G$ have a common vertex. It is proved in [11] that the line graph of all $k$-regular graphs, for $k \geq 4$, are hyperenegetic.

Let $\Gamma$ be a finite multiplicative group with identity 1 . For $S \subseteq \Gamma, 1 \notin S$ and $S^{-1}=\left\{s^{-1}: s \in S\right\}=S$, the Cayley graph $X=$ Cay $(\Gamma, S)$ is the undirected graph having vertex set $V(X)=\Gamma$ and edge set $\left\{(a, b): a b^{-1} \in S\right\}$. By the right multiplication $\Gamma$ may be considered as a group of automorphisms of $X$ acting transitively on $V(X)$. The Cayley graph $X$ is a regular graph of degree $|S|$. Its connected components are the right cosets of the subgroup generated by $S$. So $X$ is connected, if $S$ generates $\Gamma$.

For a positive integer $n>1$ the unitary Cayley graph $X_{n}=$ Cay $\left(Z_{n}, U_{n}\right)$ is defined by the additive group of the ring $Z_{n}$ of integers modulo $n$ and the multiplicative group $U_{n}$ of its units. If we represent the elements of $Z_{n}$ by the integers $0,1, \ldots, n-1$, then $U_{n}=\left\{a \in Z_{n}: \operatorname{gcd}(a, n)=1\right\}$. So, $X_{n}$ has the vertex set $V\left(X_{n}\right)=Z_{n}=\{0,1, \ldots, n-1\}$ and the edge set $\left\{(a, b): a, b \in Z_{n}\right.$, $\operatorname{gcd}(a-b, n)=1\}$.

The concept of graph energy arose in theoretical chemistry. The total $\pi$-electron energy of some conjugated carbon molecule, computed using Hückel theory, coincides with the energy of its "molecular" graph. Recently there has been a tremendous research activity in the areas like hyperenergetic graphs, maximum energy graphs, equienergetic graphs. We refer to the survey papers by Gutman [7] and by Brualdi [3] for details. The study of the energy of circulant graphs is also of number theoretic interest as it is related to the Gauss sum (see for instance [2], [9] and [10]). Cayley graphs are important class of circulant graphs defined through finite groups. The unitary Cayley graphs have number theoretic aspects as illustrated by Klotz and Sander [8] and Fuchs [5], wherein, the basic invariants, the eigenvalues and the largest induced cycles were determined.

The energy of $X_{n}$ when $n$ is a power of a prime was determined by Balakrishnan [1] using the computations involving the cyclotonic polynomials $\phi_{n}(x)$. In this note we extend the result of Balakrishnan by obtaining the energy of all unitary Cayley graphs $X_{n}$ 
and determine when they are hyperenergetic. We also obtain a lower bound for the ratio of the energy of the unitary Cayley graph and the complete graph, thus measuring the degree of hyperenergeticity. This ratio grows exponentially with the number of distinct prime factors of $n$.

\section{PRELIMINARIES}

We give a brief account of some of the results of Klotz and Sander [8] on the eigenvalues of unitary Cayley graphs which will be used in this note.

It is well known that $X_{n}$ is a connected $\phi(n)$ - regular graph. If $n=p$ is a prime number, then $X_{n}$ is the complete graph on $p$ vertices and if $n=p^{\alpha}$ is a prime power, then $X_{n}$ is a complete $p$ - partite graph. The unitary Cayley graph $X_{n}, n \geq 2$, is bipartite if and only if $n$ is even. Klotz and Sander [8] have determined the chromatic number, the clique number, the independence number, the diameter and the vertex connectivity of $X_{n}$. They have also shown that all nonzero eigenvalues of $X_{n}$ are integers dividing $\phi(n)$.

The eigenvalues of $X_{n}$ are given by

$$
\lambda_{r+1}=\sum_{\substack{1 \leq j<n, \operatorname{gcd}}} \omega^{r j}, 0 \leq r \leq n-1,
$$

where $\omega=\exp \left(\frac{2 \pi i}{n}\right)$. The sum in equation (2.1) is the well known Ramanujan sum $c(r, n)$. Thus, we have,

$$
\lambda_{r+1}=c(r, n), \quad 0 \leq r \leq n-1 .
$$

The value of $c(r, n)$ is an integer and so all the eigenvalues of $X_{n}$ are integers which are given by:

$$
c(r, n)=\mu\left(t_{r}\right) \frac{\phi(n)}{\phi\left(t_{r}\right)}, \text { where } t_{r}=\frac{n}{\operatorname{gcd}(r, n)}, \quad 0 \leq r \leq n-1,
$$

where $\mu$ denotes the Möbius function. Klotz and Sander [8] have obtained the following results:

Theorem 2.1 [8] For $n \geq 2$, the following statements hold:

1. Every nonzero eigenvalue of $X_{n}$ is a divisor of $\phi(n)$.

2. Let $m$ be the maximal squarefree divisor of $n$. Then

$$
\lambda_{\min }=\mu(m) \frac{\phi(n)}{\phi(m)}
$$

is a nonzero eigenvalue of $X_{n}$ of minimal absolute value and multiplicity $\phi(m)$. Every eigenvalue of $X_{n}$ is a multiple of $\lambda_{\min }$. If $n$ is odd, then $\lambda_{\min }$ is the only nonzero eigenvalue of $X_{n}$ with minimal absolute value. If $n$ is even, then $-\lambda_{\min }$ is also an eigenvalue of $X_{n}$ with multiplicity $\phi(m)$. 
Theorem 2.2 [8] Let $m$ be the maximal squarefree divisor of $n$ and let $M$ be the set of positive divisors of $m$. Then the following statements for the unitary Cayley graph $X_{n}, \quad n \geq 2$, hold:

1. Repeating $\phi(t)$-times every term of the sequence $S=\left(\mu(t) \frac{\phi(n)}{\phi(t)}\right)_{t \in M}$ results in a sequence $\tilde{\mathrm{S}}$ of length $m$ which consists of all nonzero eigenvalues of $X_{n}$ such that the number of appearances of an eigenvalue is its multiplicity.

2. The multiplicity of zero as an eigenvalue of $X_{n}$ is $n-m$.

3. If $\alpha(\lambda)$ is the multiplicity of the eigenvalue $\lambda$ of $X_{n}$, then $\lambda \alpha(\lambda)$ is a multiple of $\phi(n)$.

\section{ENERGY OF UNITARY CAYLEY GRAPHS}

We first give a direct proof of the result of Balakrishnan [1] when $n$ is a power of a prime.

Theorem 3.1. If $n=p^{\alpha}$ is a prime power, then the energy of the unitary Cayley graph $X_{n}$ is given by $E\left(X_{n}\right)=2 \phi(n)$.

Proof. When $\alpha=1$, the graph $X_{n}$ is the complete graph $K_{p}$. Clearly $E\left(K_{p}\right)=2(p-1)=$ $2 \phi(p)$. Hence we can assume $\alpha \geq 2$.

The eigenvalues of the unitary Cayley graph $X_{p^{\alpha}}$ are given by

$$
\lambda_{r+1}=c\left(r, p^{\alpha}\right)=\mu\left(t_{r}\right) \frac{\phi\left(p^{\alpha}\right)}{\phi\left(t_{r}\right)}, \text { where } t_{r}=\frac{p^{\alpha}}{\operatorname{gcd}\left(r, p^{\alpha}\right)}, 0 \leq r \leq p^{\alpha}-1
$$

We consider three cases:

Case(1): If $\operatorname{gcd}\left(r, p^{\alpha}\right)=p^{\alpha}$ then $r=0$ and so $t_{0}=1$. Hence $\lambda_{1}=\phi\left(p^{\alpha}\right)=p^{\alpha}-p^{\alpha-1}$.

Case(2): If $\operatorname{gcd}\left(r, p^{\alpha}\right)=1$ then $t_{r}=p^{\alpha}$ and hence we get $\lambda_{r+1}=0$.

Case(3): If $1<\operatorname{gcd}\left(r, p^{\alpha}\right)<p^{\alpha}$ then $\operatorname{gcd}\left(r, p^{\alpha}\right)=p^{m}$, where $1 \leq m \leq \alpha-1$. When $\operatorname{gcd}\left(r, p^{\alpha}\right)=p^{\alpha-1}$, we get $\lambda_{r+1}=-p^{\alpha-1}$. For all other remaining values of $m$ we get $\lambda_{r+1}=0$.

Therefore the Spectrum of $X_{p^{\alpha}}$ is

$$
\operatorname{Spec} X_{p^{\alpha}}=\left(\begin{array}{ccc}
p^{\alpha}-p^{\alpha-1} & -p^{\alpha-1} & 0 \\
1 & p-1 & p^{\alpha}-p
\end{array}\right) \text {. }
$$

Thus, $E\left(X_{p^{\alpha}}\right)=p^{\alpha}-p^{\alpha-1}+(p-1) p^{\alpha-1}=2\left(p^{\alpha}-p^{\alpha-1}\right)=2 \phi\left(p^{\alpha}\right)$.

Hence the proof.

Let $G_{1}=\left(V_{1}, E_{1}\right)$ and $G_{2}=\left(V_{2}, E_{2}\right)$ be graphs. The direct product of $G_{1}$ and $G_{2}$ is the graph $G=(V, E)$ denoted by $G_{1} \otimes G_{2}$ (also by $G_{1} \wedge G_{2}$ ) where $V=V_{1} \times V_{2}$, the 
cartesian product of $V_{1}$ and $V_{2}$, with $\left(v_{1}, v_{2}\right)$ and $\left(u_{1}, u_{2}\right)$ are adjacent in $G$ if and only if $v_{1}, u_{1}$ are adjacent in $G_{1}$ and $v_{2}, u_{2}$ are adjacent in $G_{2}$.

Theorem 3.2. If $(m, n)=1$, then the direct product of the unitary Cayley graphs $X_{m}$ and $X_{n}$ is isomorphic to $X_{m n}$.

Proof. Since $(m, n)=1$, by the Chinese Remainder theorem, there is an isomorphism $\phi: Z_{m} \times Z_{n} \longrightarrow Z_{m n}$. This isomorphism induces an isomorphism between their groups of units $U_{m} \times U_{n}$ and $U_{m n}$. Let $k_{i, j}$ be the element in $Z_{m n}$ corresponding to the element $(i, j) \in Z_{m} \times Z_{n}$. Then $(i, m)=1=(j, n)$ if and only if $\left(k_{i, j}, m n\right)=1$. The vertex set of $X_{m n}$ is $Z_{m n}$ and the vertex set of $X_{m} \times X_{n}$ is $Z_{m} \times Z_{n}$. The isomorphism $\phi$ gives the bijective correspondence between their vertex sets. Let $i_{1}$ be adjacent to $i_{2}$ in $X_{m}$ and let $j_{1}$ be adjacent to $j_{2}$ in $X_{n}$. Then $\left(i_{1}-i_{2}, m\right)=1=\left(j_{1}-j_{2}, n\right)$. Now consider $k_{i_{1}, j_{1}}, k_{i_{2}, j_{2}} \in Z_{m n}$. Since $\phi$ is an isomorphism, $k_{i_{1}-i_{2}, j_{1}-j_{2}}=k_{i_{1}, j_{1}}-k_{i_{2}, j_{2}}$. Now $\left(k_{i_{1}-i_{2}, j_{1}-j_{2}}, m n\right)=1$ and so $k_{i_{1}, j_{1}}$ and $k_{i_{2}, j_{2}}$ are adjacent in $X_{m n}$.

Conversely, if $k_{i_{1}, j_{1}}$ is adjacent to $k_{i_{2}, j_{2}}$ in $X_{m n}$, then, $k_{i_{1}-i_{2}, j_{1}-j_{2}}=k_{i_{1}, j_{1}}-k_{i_{2}, j_{2}} \in U_{m n}$ and so $i_{1}-i_{2} \in U_{m}$ and $j_{1}-j_{2} \in U_{n}$. Thus $i_{1}$ is adjacent to $i_{2}$ in $X_{m}$ and $j_{1}$ is adjacent to $j_{2}$ in $X_{n}$. Hence $X_{m} \otimes X_{n}$ and $X_{m n}$ are isomorphic. This completes the proof.

Corollary 3.3. If $n=p_{1}^{\alpha_{1}} p_{2}^{\alpha_{2}} \ldots p_{k}^{\alpha_{k}}$, then the direct product of unitary Cayley graphs $X_{p_{1}^{\alpha_{1}}} \otimes X_{p_{2}^{\alpha_{2}}} \otimes \cdots \otimes X_{p_{k}^{\alpha_{k}}}$ is isomorphic to $X_{n}$.

Definition 3.4. The tensor product $A \otimes B$ of the $r \times s$ matrix $A=\left(a_{i j}\right)$ and the $t \times u$ matrix $B=\left(b_{i j}\right)$ is defined as the $r t \times s u$ matrix got by replacing each entry $a_{i j}$ of $A$ by the double array $a_{i j} B$.

It is easy to check that for any two graphs $G_{1}$ and $G_{2}$ the adjacency matrix $A\left(G_{1} \otimes G_{2}\right)$ of $G_{1} \otimes G_{2}$ is given by

$$
A\left(G_{1} \otimes G_{2}\right)=A\left(G_{1}\right) \otimes A\left(G_{2}\right) .
$$

Lemma 3.5. [4] If $A$ is a matrix of order $r$ with Spectrum $\left\{\lambda_{1}, \lambda_{2}, \ldots, \lambda_{r}\right\}$, and $B$, a matrix of order $s$ with Spectrum $\left\{\mu_{1}, \mu_{2}, \ldots, \mu_{s}\right\}$, then the spectrum of $A \otimes B$ is $\left\{\lambda_{i} \mu_{j}: 1 \leq i \leq r ; 1 \leq j \leq s\right\}$.

Corollary 3.6. If $G_{1}$ and $G_{2}$ are any two graphs, then,

$$
E\left(G_{1} \otimes G_{2}\right)=E\left(G_{1}\right) E\left(G_{2}\right) .
$$

Theorem 3.7. If $n>1$ is of the form $n=p_{1}^{\alpha_{1}} p_{2}^{\alpha_{2}} \ldots p_{k}^{\alpha_{k}}$ where $p_{1}, p_{2}, \ldots, p_{k}$ are distinct primes and $\alpha_{1}, \alpha_{2}, \ldots, \alpha_{k}$ are positive integers, then,

$$
E\left(X_{n}\right)=2^{k} \phi(n) .
$$

Proof. By Corollary 3.3, $X_{n}$ is isomorphic to the product $X_{p_{1}^{\alpha_{1}}} \otimes \ldots \otimes X_{p_{k} \alpha_{k}}$. Now by Corollary 3.6, the energy of the direct product of graphs is the product of their energies. Hence, it follows that, $E\left(X_{n}\right)=E\left(X_{p_{1}^{\alpha_{1}}}\right) \ldots E\left(X_{p_{k}^{\alpha_{k}}}\right)$. Now by Theorem $3.1, E\left(X_{p_{i} \alpha_{i}}\right)=$ $2 \phi\left(p_{i}^{\alpha_{i}}\right)$ for $1 \leq i \leq k$ and so we have, 


$$
\begin{aligned}
E\left(X_{n}\right) & =2^{k} \phi\left(p_{1}^{\alpha_{1}}\right) \ldots \phi\left(p_{k}^{\alpha_{k}}\right) \\
& =2^{k} \phi\left(p_{1}^{\alpha_{1}} \ldots p_{k}^{\alpha_{k}}\right) \\
& =2^{k} \phi(n), \text { since } \phi \text { is multiplicative. }
\end{aligned}
$$

Corollary 3.8. $\frac{E\left(X_{n}\right)}{2(n-1)}>2^{k-1} \frac{\phi(n)}{n}$.

We note that if $n=p_{1}^{\alpha_{1}} p_{2}^{\alpha_{2}} \ldots p_{k}^{\alpha_{k}}$, then,

$$
\frac{\phi(n)}{n}=\left(1-\frac{1}{p_{1}}\right)\left(1-\frac{1}{p_{2}}\right) \ldots\left(1-\frac{1}{p_{k}}\right) .
$$

This will be used in the characterization of hyperenergetic unitary Cayley graphs. First we state the following Lemma whose proof follows by induction and is elementary.

Lemma 3.9. For $k \geq 3$ and $n=p_{1}^{\alpha_{1}} p_{2}^{\alpha_{2}} \ldots p_{k}^{\alpha_{k}}$, we have,

$$
\frac{\phi(n)}{n}>\frac{1}{2^{k-1}}
$$

By making use of the Theorem 3.7, we now characterise the hyperenergetic unitary Cayley graphs.

Theorem 3.10 Let $n=p_{1}^{\alpha_{1}} p_{2}^{\alpha_{2}} \ldots p_{k}^{\alpha_{k}}$ where $p_{1}, p_{2}, \ldots, p_{k}$ are distinct prime divisors of $n$. Then the unitary Cayley graph $X_{n}$ is hyperenergetic if and only if $k \geq 3$ or $k=2$ and $n$ is odd.

Proof. We consider three cases:

Case 1: For $k=1, n=p^{\alpha}$, if $X_{n}$ is hyperenergetic then, we have,

$$
2 \phi\left(p^{\alpha}\right)>2\left(p^{\alpha}-1\right) \Rightarrow 2\left(p^{\alpha}-p^{\alpha-1}\right)>2\left(p^{\alpha}-1\right)
$$

i.e., $\quad 2>2 p^{\alpha-1} \Rightarrow 1>p^{\alpha-1}$

which is impossible.

Therefore $X_{n}$ is not hyperenergetic.

Case 2: For $k=2, n=p^{\alpha} q^{\beta} \quad(p<q)$.

Here we consider two subcases:

(i) $p=2, n=2^{\alpha} q^{\beta}, 2<q$

Then, we have,

$$
\begin{aligned}
E\left(X_{n}\right)=4 \phi(n)=4 & \cdot 2^{\alpha-1} q^{\beta-1}(q-1) \\
= & 2 n\left(\frac{q-1}{q}\right)<2 n\left(\frac{n-1}{n}\right)=2(n-1) .
\end{aligned}
$$

Therefore $X_{n}$ is non-hyperenergetic. 
(ii) $p \geq 3, q \geq 3, \quad p<q$

Since $q \geq 5$, we have, $E\left(X_{n}\right)>2 n$.

Therefore $X_{n}$ is hyperenergetic.

Case 3: Let $k \geq 3$. If $n=p_{1}^{\alpha_{1}} p_{2}^{\alpha_{2}} \ldots p_{k}^{\alpha_{k}}$, then, by Corollary 3.8 and Lemma 3.9, we have,

$$
\frac{E\left(X_{n}\right)}{2(n-1)}>1
$$

and so $X_{n}$ is hyperenergetic.

This completes the proof of the theorem.

In the next theorem we show that the degree of hyperenergeticity grows at least exponentially with the number of distinct prime divisors of $n$ by making use of the sharper lower bound for $\frac{\phi(n)}{n}$, namely $\frac{\phi(n)}{n}>\frac{1}{2 k}$.

Theorem 3.11. Let $k$ denote the number of distinct prime divisors of $n$. Then

$$
\frac{E\left(X_{n}\right)}{2(n-1)}>\frac{2^{k}}{4 k}
$$

Proof. Let $n=q_{1}^{\alpha_{1}} \ldots q_{k}^{\alpha_{k}}$ where $q_{1}, \ldots, q_{k}$ are distinct primes such that $q_{1}<q_{2}<\ldots<q_{k}$. When $k=1$, we have, $n=q^{\alpha}$ and so

$$
\frac{E\left(X_{n}\right)}{2(n-1)}=\frac{2 \phi(n)}{2(n-1)}>\frac{\phi(n)}{n}=\left(1-\frac{1}{q}\right) \geq \frac{1}{2} .
$$

Suppose $k \geq 2$. Let $p_{j}$ denote the $j^{\text {th }}$ prime. Then clearly $p_{j} \geq 2 j-1$ for $j \geq 2$. Thus

$$
1-\frac{1}{q_{j}} \geq 1-\frac{1}{p_{j}} \geq \frac{2 j-2}{2 j-1}
$$

Hence,

$$
\frac{\phi(n)}{n} \geq \frac{1}{2} \cdot \frac{2}{3} \cdot \frac{4}{5} \cdot \frac{6}{7} \cdots \frac{2 k-2}{2 k-1} \geq \frac{1}{2 k-1}>\frac{1}{2 k} .
$$

Now the result follows from Corollary 3.8.

\section{Acknowledgement}

The authors thank the referee for helpful comments and useful suggestions. 


\section{References}

[1] R. Balakrishnan, The energy of a graph, Linear Algebra Appl. 387 (2004) 287-295.

[2] S.R. Blackburn, I. Shparlinski, On the average energy of circulant graphs, Linear Algebra Appl. 428 (2008) 1956-1963.

[3] R.A. Brualdi, Energy of a graph, Notes to AIM Workshop on spectra of families of matrices described by graphs, digraphs, and sign patterns, 2006.

[4] D. Cvetković, M. Doob, H. Sachs, Spectra of Graphs - Theory and Applications, Academic Press, New York, 1980.

[5] E.D. Fuchs, Largest induced cycles in circulant graphs, The Electronic Journal of Combinatorics, 12 (2005), 1-12.

[6] I. Gutman, The energy of a graph, Ber. Math. -Stat. Sekt. Forschungszent. Graz 103 (1978) 1-22.

[7] I. Gutman, The energy of a graph: old and new results in : A. Bitten, A. Koher, R. Laue and A. Wassermann (Eds.), Algebraic Combinatorics and Applications, Springer-Verlag, Berlin 2001, 196-211.

[8] W. Klotz, T. Sander, Some properties of unitary Cayley graphs, The Electronic Journal of Combinatorics, 14 (2007) 1-12.

[9] I. Shparlinski, On the energy of some circulant graphs, Linear Algebra and Appl., 414 (2006), 378-382

[10] D. Stevanonic and I. Stankovic, Remarks on hyperenergetic circulant graphs, Linear Algebra and Appl., 400 (2005), 345-348.

[11] H.B. Walikar, I. Gutman, P.R. Hampliholi, H.S. Ramane, Non-hyperenergetic graphs, Graph Theory Notes N.Y. 51 (2001) 14-16. 